\title{
Endobronchial presentation of Hodgkin's disease: a report of nine cases and review of the literature
}

\author{
J. Trédaniel*, I. Peillon*, C. Fermé**, P. Brice**, \\ C. Gisselbrecht**, A. Hirsch*
}

Endobronchial presentation of Hodkgin's disease: a report of nine cases and review of the literature. J. Trédaniel, I. Peillon, C. Fermé, P. Brice, C. Gisselbrecht, A. Hirsch. (c) ERS Journals Ltd 1994.

ABSTRACT: Although mediastinal involvement by Hodgkin's disease is frequent, the initial presentation of the lymphoma by an endobronchial lesion is rare. Therefore, the question of whether patients with Hodgkin's disease should undergo fibreoptic bronchoscopy as a staging procedure remains unresolved.

In a series of 469 patients with newly diagnosed Hodgkin's disease during a 10 year period, we reviewed the clinical features of nine patients who presented with an endobronchial tumour. They were compared to 34 previously published cases.

The major presenting symptoms were cough, wheezing and haemoptysis. Bulky mediastinum was seen in six cases. The three other patients presented respiratory symptoms evocative of endoluminal invasion. All but one of the patients received combined modality therapy, as currently accepted for patients with poor prognostic factors. The overall actuarial survival was $74 \%$ after $4 \mathrm{yrs}$ of follow-up.

A selective subgroup of patients with stage I-II supradiaphragmatic and endobronchial Hodgkin's disease may, thus, present without poor prognostic factors (but generally with respiratory symptoms), and might be undertreated if this localization is not recognized. We propose that these patients should undergo fibreoptic bronchoscopy.

Eur Respir J., 1994, 7, 1852-1855. \author{
Louis, Paris France. \\ Correspondence: J. Trédaniel \\ Service de Pneumologie \\ Hôpital Saint-Louis \\ 1 Ave Claude Vellefaux \\ 75475 Paris cedex 10 \\ France
}

*Service de Pneumologie and**Département d'Onco Hématologie, Hôpital Saint-

Keywords: Bronchoscopy
endobronchial localization

Hodgkin's disease

Received: January 241994

Accepted after revision June 181994
The mediastinum is involved in the majority of patients with newly diagnosed Hodgkin's disease, most commonly in those with nodular sclerosing histological subtype [1]. Radiographic manifestations on chest X-ray typically include bilateral but asymmetrical mediastinal and hilar lymphadenopathy. Mediastinal adenopathy commonly occurs in the paratracheal and bifurcation lymph nodes, followed closely by the bronchopulmonary nodes. Evidence of intrathoracic lymph node involvement is seen in up to $85 \%$ of patients using chest computed tomography (CT) [2]. Tracheobronchial compression is quite common in children [3], but extremely rare in adults, because lymph nodes are soft relative to the cartilaginous skeleton of the trachea and proximal bronchi. Thus, although Hodgkin's disease in the mediastinum occurs frequently, symptoms characteristic of the disease at this site are less common [4].

Presentation of Hodgkin's disease as an endobronchial lesion is very uncommon. Due to respiratory symptoms, which can be predominant in these cases, the pulmonologist may be confronted with the diagnosis of this condition. Unrecognized, it can lead to an understaging of the disease that can compromise the cure rate. However, the experience of individual practitioners with this presentation of Hodgkin's disease is obviously limited. We had the opportunity to observe nine cases of Hodgkin's disease presenting as an endobronchial tumour, over a 10 year period. A retrospective analysis of these patients, including a comparison with previously published cases, was undertaken in order to define the role of bronchoscopy in the initial evaluation of patients with Hodgkin's disease.

\section{Material and methods}

From 1982 to 1992,469 patients (mean age 35 y) with newly diagnosed Hodgkin's disease were treated in four consecutive protocols, at the Hôpital Saint-Louis in Paris, France $[5,6]$. Two hundred and twenty five patients were males (48\%), mean age 35 yr.) Initial staging procedures included physical examination, complete blood cell count and routine blood chemistry, posteroanterior and lateral chest roentgenograms, bipedal lymphangiography, upper abdominal examination by echography or CT scan, and bone marrow biopsy. Thoracic CT was routinely used after 1984 . A total of 399 patients $(85 \%)$ showed evidence of thoracic Hodgkin's disease on their chest radiographs or CT scans. At presentation, bulky mediastinal involvement was defined as a ratio between the largest mediastinal mass diameter and the widest transverse diameter of the chest $>0.33$ [7]. Sixty one patients $(13 \%)$ fulfiled the criteria of bulky mediastinum. Other tests, such as endoscopy, were performed when clinically indicated.

Staging evaluation was performed according to the 
Table 1. - Clinical data and outcome of nine patients with Hodgkin's disease presenting with an endobronchial tumour

\begin{tabular}{|c|c|c|c|c|c|c|c|c|c|}
\hline Case No. & 1 & 2 & 3 & 4 & 5 & 6 & 7 & 8 & 9 \\
\hline Sex & $\mathrm{F}$ & M & M & $\mathrm{F}$ & $\mathrm{F}$ & M & M & M & $\mathrm{F}$ \\
\hline Age yrs & 28 & 27 & 83 & 52 & 39 & 34 & 29 & 21 & 26 \\
\hline Cough & Yes & Yes & Yes & No & No & Yes & No & Yes & Yes \\
\hline Wheezing & No & No & No & No & No & No & Yes & No & No \\
\hline Haemoptysis & No & No & No & No & No & Yes & No & No & No \\
\hline Bulky mediastinum & Yes & Yes & No & Yes & Yes & No & No & Yes & Yes \\
\hline Bronchoscopic Location & $\mathrm{R}$ & $\mathrm{R}$ & $\mathrm{R}$ & $\mathrm{T}, \mathrm{R}, \mathrm{L}$ & $\mathrm{R}$ & $\mathrm{L}$ & $\mathrm{R}$ & $\mathrm{R}$ & $\mathrm{R}$ \\
\hline Extrathoracic extention & Bone & No & No & No & Abdominal & No & No & Bone & Abdominal \\
\hline Histological subtype & NS & MC & MC & LD & NS & NS & $\mathrm{MC}$ & NS & NS \\
\hline Ann Arbor stage & $\mathrm{IV} \mathrm{Bb}$ & $\mathrm{IV} \mathrm{Bb}$ & $\mathrm{IV} \mathrm{Bb}$ & IV $\mathrm{Ab}$ & IV $\mathrm{Ab}$ & $\mathrm{IV} \mathrm{Bb}$ & IV Aa & $\mathrm{IVBb}$ & IV $\mathrm{Bb}$ \\
\hline Clinical outcome & $\mathrm{CR}$ & Died & Died & $\mathrm{CR}$ & $\mathrm{CR}$ & $\mathrm{CR}$ & $\mathrm{CR}$ & $\mathrm{CR}$ & $\mathrm{CR}$ \\
\hline Duration of follow-up & $96 \mathrm{~m}$ & $45 \mathrm{~m}$ & $6 \mathrm{~m}$ & $84 \mathrm{~m}$ & $66 \mathrm{~m}$ & $66 \mathrm{~m}$ & $48 \mathrm{~m}$ & $20 \mathrm{~m}$ & $15 \mathrm{~m}$ \\
\hline
\end{tabular}

F: female; M: male; R: right bronchial tree; L: left bronchial tree; T: trachea; NS: nodular sclerosis; MC: mixed cellularity; LD: lymphocyte depletion; CR: complete response; m: month.

Ann Arbor classification. All pathological diagnoses were made by specialized pathologists. The histological subtypes were: lymphocyte predominance 24 patients $(5 \%)$; nodular sclerosis 332 patients $(71 \%)$; mixed cellularity 89 patients (19\%); lymphocyte depletion 11 patients (2\%); and unclassified 13 patients $(3 \%)$.

To be recognized as endobronchial presentation of Hodgkin's disease, the cases had to fulfil the following criteria: 1) histological features of Hodgkin's disease (wherever the site of biopsy); and 2) bronchoscopic visualization of an endobronchial tumour, at the time of the initial diagnosis of the disease. The following features were reviewed: age, sex, clinical and radiological presentation, bronchoscopic appearance and location, and long-term outcome. The survival of these patients was estimated from the time of pathological diagnosis, using the actuarial method.

The previous data available were identified in the international literature from 1970 by Medline searches on endobronchial Hodgkin's disease involvement. Earlier references were identified from citations in the more recent papers and reviews (references up to 1980 are available by request to the first author).

\section{Results}

Nine cases of Hodgkin's disease, fulfiling the criteria described above, were diagnosed and treated during the period 1982-1992. Clinical details of patients are summarized in table 1 . The major presenting symptom was cough. One patient each complained of wheezing or haemoptysis. No patient had dyspnoea. Two patients did not present any respiratory symptoms, and bronchoscopy was decided upon because of bulky mediastinum. Only one patient presented recent wheezing, for which a chest X-ray was performed. Mediastinum was involved in all patients, and was found to be bulky in six cases. Chest X-ray showed the presence of atelectasis in two patients. One patient each presented with pleural effusion and pulmonary parenchymal nodules. Other manifestations included: supraclavicular and cervical nodes (7); systemic symptoms (6); bone involve- ment (2); one case with neurological complication; and para-aortic extension (2).

All nine patients showed endoluminal invasion of the respiratory tree by a lymphoid tumour mass. The trachea was involved in one case, the right bronchial tree in eight cases, and the left bronchial tree in two cases. One patient, although asymptomatic (case No. 4), presented a huge endoluminal tumour mass extending from the trachea into the main bronchi. In all patients, the lesion had the appearance of a polypoid mass projecting into the lumen. Endobronchial biopsies were conclusive of Hodgkin's disease in four patients, with similar histological appearance to that of the final diagnostic specimens (mainly peripheral lymph nodes), but were only indicative of Hodgkin's disease in four cases. In one patient (case No. 3) the appearance of the endobronchial specimens was inconclusive, and the diagnosis was obtained after mediastinoscopy; the probability that the endobronchial lesion was due to other disease was very low, and this patient was considered to fulfil our inclusion criteria.

Excluding the oldest patient, all patients were treated as stage IV disease with combination chemotherapy and radiotherapy. Radiation therapy was delivered using mantle fields in all cases, and additional subdiaphragmatic irradiation in seven cases. Two patients died, 6 and 45 months after initial diagnosis; the patient who died after 6 months was 83 yrs of age at diagnosis and could not receive any other treatment than low-dose chemotherapy. The second patient died after familial bone marrow transplantation, without having obtained a complete remission. The other patients remain in their first complete remission after 15-96 months of followup. The median duration of follow-up is 4 yrs. The overall survival of the group is $74 \%$ after 4 yrs and then maintains a plateau.

\section{Discussion}

Mediastinal involvement is very common in Hodgkin's disease $[2,8]$. Based on autopsy data, the incidence of intrabronchial involvement in cases of established 
Hodgkin's disease may be as high as $15 \%$, but most frequently develops during the disease. Initial endobronchial involvement is very rare; only 34 cases, which fulfilled our inclusion criteria, were found in the literature [9-16]. However, many of these were published a long time ago, and precise data are lacking. Clinical details of these cases, when available, are summarized in table 2 . Of the 34 cases of endobronchial presentation of Hodgkin's disease, haemoptysis occurred in 10; haemoptysis is an unusual occurrence in Hodgkin's disease without this presentation. Dyspnoea and coughing were the most frequent symptoms. Cases of life-threatening obstruction have been described [15]. Enlarged hilar lymph nodes were present radiologically in 21 cases (but chest X-ray appeared to be normal in three cases). The lesion was bronchoscopically described under the form of plaquelike infiltrates of the mucosa with superficial ulceration, or a single polypoid or adenomatous mass projecting into the lumen [12]. In 17 patients, the diagnosis was made by bronchoscopic biopsy.

The incidence of endobronchial presentation of Hodgkin's disease is difficult to state, but could be more frequent than previously thought. In a small series of patients who underwent systematic fibreoptic bronchoscopy during initial staging, 3 of 12 cases had pathologically proven endobronchial lymphoma [11]. In our study, bronchoscopy was not performed in a systematic prospective fashion but only when respiratory symptoms or bulky mediastinum was present. Therefore, it is possible that we underestimated cases of asymptomatic endobronchial disease because of our retrospective study design.

Our description of nine patients with endoluminal lymphoma at presentation adds to the previously published

Table 2. - Clinicopathological characteristics of 34 patients presenting with an endobronchial localization of Hodgkin's disease at the time of diagnosis

\begin{tabular}{lc}
\hline Age $(\mathrm{n}=31)$ yrs* & $37 \pm 16$ \\
& $(15-75)$ \\
Sex $(\mathrm{n}=31) \quad$ M/F & $17 / 14$ \\
Respiratory signs at presentation (n=31) & \\
Dyspnoea & 21 \\
Coughing & 19 \\
Wheezing & 10 \\
Haemoptysis & 10 \\
Sputum production & 5 \\
Chest pain & 3 \\
Chest X-ray findings (n=33) & 3 \\
Normal chest X-ray & 21 \\
Mediastinal widening & 8 \\
Atelectasis & 2 \\
Pleural effusion & \\
Location of the tumour at bronchoscopy & 11 \\
Trachea & 16 \\
Right bronchial tree & 10 \\
Left bronchial tree & 17 \\
Bronchial biopsy positive or suggestive & \\
Histiological subtype (n=12) & 6 \\
Mixed cellularity & 5 \\
Nodular sclerosis & 1 \\
Lymphocyte depletion &
\end{tabular}

Data were not available for all patients. *: mean \pm SD and range in parenthesis. M: male; F: female. [9-16] cases on this rare clinical situation. During the period under study, 469 new cases of Hodgkin's disease were seen, giving a $2 \%$ incidence of lymphoid airway involvement. Six of our patients had bulky mediastinum, as compared to 61 patients of the total population (13\%). All except one of our cases received combined modality treatment, and their overall survival corresponds well with what has been published previously [17].

In Hodgkin's disease, careful staging and optimal treatment can lead to cure for approximately $75 \%$ of patients [18]. For stages I-II supradiaphragmatic Hodgkin's disease, the selection of treatment is above all dependent on the presence of poor prognostic factors $[19,20]$. In particular, it is now recognized that conventional therapy for any Hodgkin's patient who presents with poor prognostic factors, especially bulky mediastinal disease, is the use of combination chemotherapy and radiotherapy $[21,22]$. Therefore, primary endobronchial Hodgkin's disease, which is most often associated with bulky mediastinum, is now treated by combined modality therapy. However, our data demonstrates that a very selective subgroup of patients with early supradiaphragmatic disease, without poor prognostic factors, may be treated by radiotherapy alone even if an undiagnosed endobronchial lesion is present. We suggest that these patients be considered for bronchoscopy, if they present with respiratory symptoms. A prospective study would be of interest to determine whether bronchoscopy should be included in staging for Hodgkin's disease.

\section{References}

1. Colby TV, Hoppe RT, Warnke RA. Hodgkin's disease: a clinicopathological study of 659 cases. Cancer 1981; 49: 1848-1858.

2. Castellino RA, Blank N, Hoppe RT, Cho C. Hodgkin's disease: contributions of chest CT in the initial staging evaluation. Radiology 1986; 160: 603-605.

3. Mandell GA, Lantieri R, Goodman LR. Tracheobronchial compression in Hodgkin's lymphoma in children. Am J Radiol 1982; 139: 1167-1170.

4. Berkman N, Breuer R. Pulmonary involvement in lymphoma. Respir Med 1993; 87: 85-92.

5. Fermé C, Brice P, Bourstyn E, et al. Surgical restaging of advanced Hodgkin's disease after first line chemotherapy. Eur J Haematol 1991; 46: 306-311.

6. Fermé C, Lepage E, D'agay MF, et al. Hodgkin's disease, clinical stages I, II A-B and III-A. Results of brief chemotherapy followed by irradiation. Nouv Rev $\mathrm{Fr}$ Hématol 1992; 34: 247-255.

7. Mauch P, Goodman R, Hellman S. The significance of mediastinal involvement in early stage Hodgkin's disease. Cancer 1978; 42: 1039-1045.

8. Diehl LF, Hopper KD, Giguere J, Granger E, Lesar M. The pattern of intrathoracic Hodgkin's disease assessed by computed tomography. J Clin Oncol 1991; 9: 438-443.

9. Lupo A, Baglione G, Lovato C, Perino B, Cardesi E. Il morbo di Hodgkin endotoracico. Descrizione di tre casi a prevalente estrinsecazione endobronchiale. Min Med 1980; 71: 419-424.

10. Kueh YK, Teoh PC. Endobronchial Hodgkin's disease; A diagnostic dilemma. Ann Acad Med Singapore 1982; 11: 294-296.

11. Gallagher CJ, Knowles GK, Habeshaw JA, Green M, Malpas JS, Lister TA. Early involvement of the bronchi 
in patients with malignant lymphoma. Br J Cancer 1983; 48: 777-781.

12. Harper PG, Fisher C, MC Lennan K, Souhami RL. Presentation of Hodgkin's disease as an endobronchial lesion. Cancer 1984; 53: 147-150.

13. Muretto P, Ugolini M, Grianti C, Fabbretti G. Tracheal Hodgkin's disease. Report of a case. Tumouri 1984; 70: 109-111.

14. Davidson NGP. Tracheal infiltration causing stridor: a rare presentation of stage I Hodgkin's disease. $\mathrm{Br} \mathrm{J} \mathrm{Clin}$ Pract 1990; 44: 677-678.

15. Jeffery GM, Mead GM, Whitehouse JMA. Life-threatening airway obstruction at the presentation of Hodgkin's disease. Cancer 1991; 67: 506-510.

16. Tanaka H, Nakahara K, Sakai S, Mimoto H, Tomita R, Gotoh K. A case of Hodgkin's disease with endotracheal tumour presenting with severe airflow obstruction. Nippon Kyobu Shikkan Gakkai Zasshi 1992; 30: 1732-1737.

17. Longo DL, Young RC, Wesley M, et al. Twenty years of MOPP therapy for Hodgkin's disease. J Clin Onco 1986; 4: 1295-1306.
18. De Vita VT, Hubbard SM. Hodgkin's disease. N Engl J Med 1993; 328: 560-565.

19. Mauch P, Tarbell N, Wienstein H, et al. Stage IA and IIA supradiaphragmatic Hodgkin's disease: prognostic factors in surgically staged patients treated with mantle and para-aortic irradiation. J Clin Oncol 1988; 6: 1576-1583.

20. Tubiana M, Henry-Amar M, Carde P, et al. Toward comprehensive management tailored to prognostic factors of patients with clinical stages I and II in Hodgkin's disease. The EORTC Lymphoma Group Controlled Clinical Trials: 1964-1987. Blood 1989; 73: 4756.

21. Hoppe RT, Coleman CN, Cox RS, Roseenberg SA, Kaplan HS. The management of stage I-II Hodgkin's disease with irradiation alone or combined modality therapy: the Stanford experience. Blood 1982; 59: 455-465.

22. Longo DL, Russo A, Duffy PL, et al. Treatment of advanced-stage massive mediastinal Hodgkin's disease: the case for combined modality treatment. J Clin Oncol 1991; 9: 227-235. 